\title{
Lung fibrosis, bone marrow fibrosis and liver cirrhosis: A Short Telomere Syndrome or a casual association?
}

\author{
Lucia Carulli $^{1}$, Claudia Anzivino ${ }^{1}$, Marco Bertolotti ${ }^{1}$, Paola Loria ${ }^{1}$, Luca Richeldi ${ }^{2}$, Stefania Cerri ${ }^{3}$ \\ 1. Department of Biomedical, Metabolic and Neural Sciences, University of Modena and Reggio Emilia, I taly. 2. Respiratory \\ Biomedical Research Unit, University of Southampton, UK. 3. Center for Rare Lung Disease, Department of Medical and \\ Surgical Sciences for Children \& Adults. University of Modena and Reggio Emilia, Italy.
}

Correspondence: Lucia Carulli. Address: Department of Biomedical, Metabolic and Neural Sciences, University of Modena and Reggio Emilia, Modena and Reggio Emilia, Italy. Email: lucia.carulli@unimore.it

Received: July 27, 2014

DOI : $10.5430 /$ crim.v2n1p11
Accepted: August 20, $2014 \quad$ Online Published: September 12, 2014

URL: http://dx.doi.org/10.5430/crim.v2n1p11

\section{Abstract}

Background: Telomere-mediated disease has diverse presentations that span the age spectrum. Their type, age of onset, and severity depend on the extent of the telomere length defect. During adult life, telomerase mutations may represent risk factors rather than genetic determinants and need other factors to contribute to disease development. This is case of diseases such as aplastic anemia, pulmonary fibrosis and liver cirrhosis which may occur as single disease or together in a syndromic clustering. Here we report a case of a man most likely affected by a short telomere syndrome.

Case report: A 58 years old man, presented for evaluation of pulmonary fibrosis diagnosed few years earlier in a different medical center. He also presented a mild bone marrow fibrosis and a liver cirrhosis, both diagnosed one year prior evaluation with a bone marrow analysis and liver biopsy. The patient was an active smoker, obese, with digital clubbing and inspiratory Velcro crackles at the right lower lobe. Laboratory tests showed thrombocytopenia and liver enzymes alteration. He rapidly developed ascites and progression of the pulmonary fibrosis, the patient became oxygen-dependent in few months.

Methods: Sequencing and mutation analysis of hTERT and hTERC genes, Leukocyte Telomere length (LTL) and Telomerase activity (TA) were evaluated.

Results: In our patient LTL was shorter and TA reduced compared to the controls. Genes sequencing did not show any hTERT and hTERC mutations.

Conclusions: This is a report on a short telomere syndrome involving lung, liver and bone marrow, associated to very short telomere and absent telomerase activity not in the setting of dyskeratosis congenita.

The fact that short telomeres mediate inflammation and fibrosis provides a rationale for pursuing translational strategies aimed at preventing telomere shortening or its cellular consequences as a therapeutic approach.

\section{Keywords}

Telomere, Telomerase, Lung fibrosis, Liver cirrhosis, Bone marrow fibrosis 


\section{Introduction}

Telomeres consist of repetitive DNA sequences (TTAGGG) associated with a specialized protein complex named shelterin and are located at the ends of linear chromosomes. They function as a cap to stabilize and protect chromosomes from erosion and from being mistaken for double- strand DNA breaks ${ }^{[1]}$. During each cell division, telomeres shorten due to the "end-replication problem" that is the DNA polymerase's inability to fully replicate the 3' end of chromosomes. In order to limit telomere attrition, germline and some somatic cells express telomerase, a reverse transcriptase that maintains telomere length by synthesizing new DNA sequences and adding them to the end of the chromosome ${ }^{[2]}$. Telomerase is an enzymatic protein complex including the telomerase reverse transcriptase (TERT) and the telomerase RNA component (TERC) used as a template to synthesize telomere DNA. Mutations in the hTERT and hTERC genes are considered the most common cause of inherited human telomere-mediated disease ${ }^{[3]}$. Even with a mild reduction in telomerase activity, telomere length homeostasis may be altered and results in what has been called a syndrome complex which include different age-dependent disease ${ }^{[4,5]}$. Telomere-mediated disease has diverse presentations that span the age spectrum. Their type, age of onset, and severity depend on the extent of the telomere length defect ${ }^{[6]}$.

Telomerase mutations may have high penetrance and induce, during infancy, severe telomere shortening that manifests with the rare Hoyeraal-Hreidarsson syndrome (developmental delay, cerebellar hypoplasia, immunodeficiency) ${ }^{[7,8]}$.

In children and young adults, they may cause the mucocutaneous syndrome dyskeratosis congenita, which is defined by a triad of mucocutaneous features-skin hyperpigmentation, dystrophic nails, and oral leukoplakia ${ }^{[9-11]}$.

During adult life, telomerase mutations may represent risk factors rather than genetic determinants and need environmental, epigenetic or other genetic factors to contribute to disease development. These mutations are less penetrant and induce single or multiple-organs damage in adults, usually without the classic physical signs of dyskeratosis congenita. This is case of diseases such as aplastic anemia, pulmonary fibrosis and liver cirrhosis which may occur as single disease or together in a syndromic clustering.

Moreover mutant telomere genes may also cause subclinical diseases in other organs even if one single organ disease manifestations dominate. For example patient with IPF who have a telomerase mutation is at increased risk to develop liver diseases as well as bone marrow failure ${ }^{[12,13]}$. It has been proposed that their shared short telomere length defect unifies them as a single syndrome continuum ${ }^{[14]}$.

Taken together the data from the literature suggest that short telomere length is associated, in general, with a more severe disease progression and a worst prognosis in several chronic diseases. Nonetheless, at present telomere length cannot be considered a diagnostic or prognostic tool in common clinical practice.

Here we report a case of a man most likely affected by a short telomere syndrome.

\section{Case presentation}

A 58 years old man, presented for evaluation of pulmonary fibrosis diagnosed few years earlier in a different medical center. At the time of presentation he reported progressive dyspnea on exertion and dry cough. Pulmonary function tests excluded the presence of significant obstructive and restrictive ventilatory impairment, but showed a moderate-to- severe reduction in diffusing capacity (DLCO). The high-resolution chest CT scan showed a diffuse infiltrative lung disease characterized by predominant ground glass opacities, traction bronchiectasis and minimum honeycombing, without definite apical-basal gradient and with relative sparing of the lung bases; concomitant centrilobular emphysema in the upper lobes was also present (see Figure 1). He was an active smoker (10 pack-years), with no history of drugs or toxic substances exposure, he also denied alcohol intake. He worked as garbage collector. 


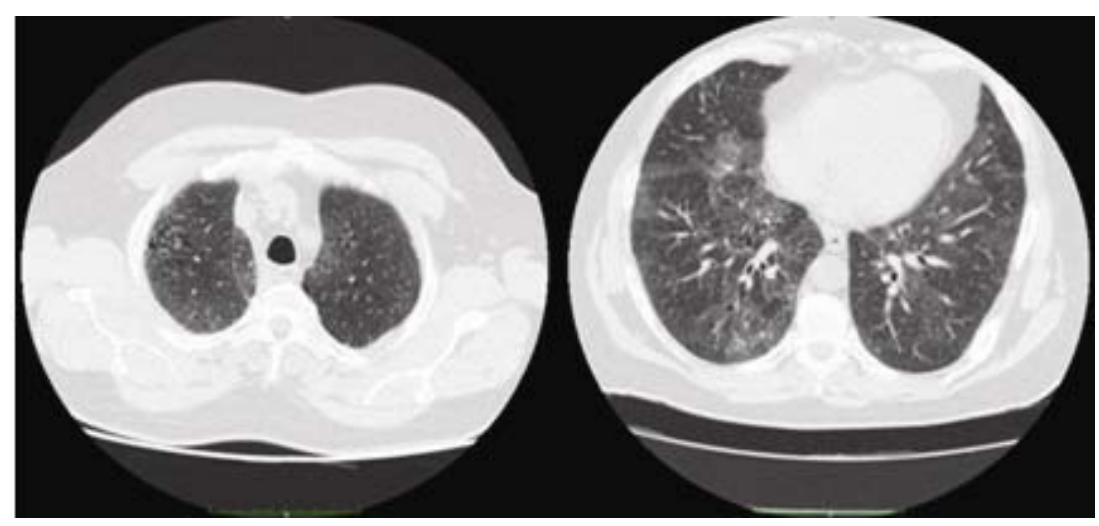

Figure 1. Chest hrCT scan. It shows the high resolution CT scan of the chest. It shows a diffuse interstitial lung disease characterized by predominant ground glass opacities (Panels A and B), with centrilobular emphysema in the upper lobes (Panel A) and traction bronchiectasis (Panel B).

His past medical history was significant for a mild bone marrow fibrosis and a non-alcoholic steatohepatitis (NASH)cirrhosis both diagnosed one year prior evaluation. The bone marrow fibrosis was diagnosed with bone marrow analysis (see Figure 2) performed for the occasional observation at routine laboratory tests of thrombocytopenia and after the observation of chronic hepatopathy with a mild splenomegaly at the abdominal ultrasound.

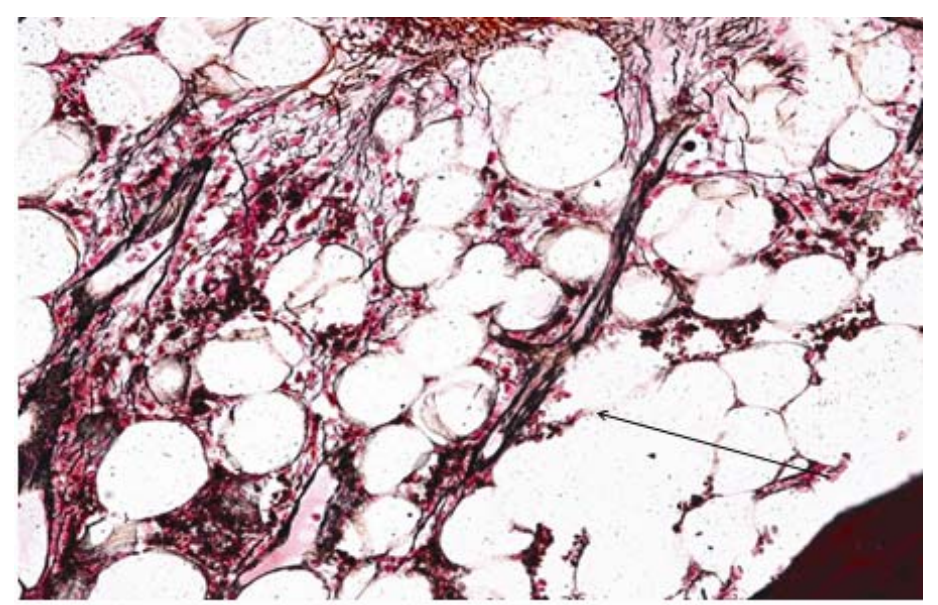

Figure 2. Bone marrow histology. As shown in Figure 2, the hematoxylin-eosin staining of bone marrow biopsy shows a mild fibrosis indicated by the black arrow pointing to branches of fibrosis.

After the abdominal US and the exclusion of all known etiologies of chronic liver disease (viral hepatitis serology, tests for autoimmunity, alpha1-antitrypsin, iron status, ceruloplasmin, serum and urinary copper) the patient underwent liver biopsy and NASH - Cirrhosis was diagnosed.

His family history was also quite interesting: his mother, aged 79, was overweight and affected by type 2 diabetes, nonalcoholic fatty liver disease (NAFLD) and was also diagnosed with colon adenocarcinoma surgically treated in the past. His father died the age of 54 with pulmonary adenocarcinoma. He had two brothers: one aged 47 with gallstone disease and the other aged 50 years with NAFLD and obesity.

On examination the patient presented obesity (BMI $32.8 \mathrm{~kg} / \mathrm{m}^{2}$ ), digital clubbing (which was known since few years earlier) and inspiratory Velcro crackles at the right lower lobe. Laboratory tests showed thrombocytopenia and liver enzymes alteration. Oxygen saturation while breathing room air at rest was $94 \%$. The follow-up was characterized by Published by Sciedu Press 
worsening of liver function with development of ascites and by a rapid progression of the pulmonary fibrosis, the patient became oxygen-dependent in few months. One year after the first evaluation the patient was still alive, but the clinical picture was markedly worsened. Subsequently the patient was lost at follow up.

The clinical presentation of this patient was quite suggestive of a short telomere syndrome. So we decided to evaluate leukocyte telomere length (LTL), telomerase activity and the presence of mutations in hTERT and hTR genes.

\section{Methods}

\subsection{Sequencing and mutation analysis and Leukocyte Telomere length assay}

Sequencing of both hTERT and hTERC genes and telomere length assay were performed as previously described ${ }^{[15]}$. Patient's LTL was compared to LTL of a group of healthy subjects ( $n=80$, age $=24-60 \mathrm{yrs}$ ). The patients and the control subjects gave their informed consent prior participation to the study.

\subsection{Telomerase activity}

Telomerase activity (TA) assay was detected with a photometric-enzyme immunoassay for the detection of telomerase activity, utilizing the Telomeric Repeat Amplification Protocol (TRAP) (TeloTAGGG Telomerase PCR Elisa -ROCHE). Patient's telomerase activity was compared to the TA of a group of healthy subjects ( $\mathrm{n}=27$, age $=24-60 \mathrm{yrs}$ ).

\section{Results}

\subsection{Leukocyte Telomere length}

The Scatter Blot (see Figure 3) shows the leukocyte telomere length distribution in relation to age in our patient and in the group of healthy subjects. The LTL in our patient was greatly shorter compared to age matched healthy controls.

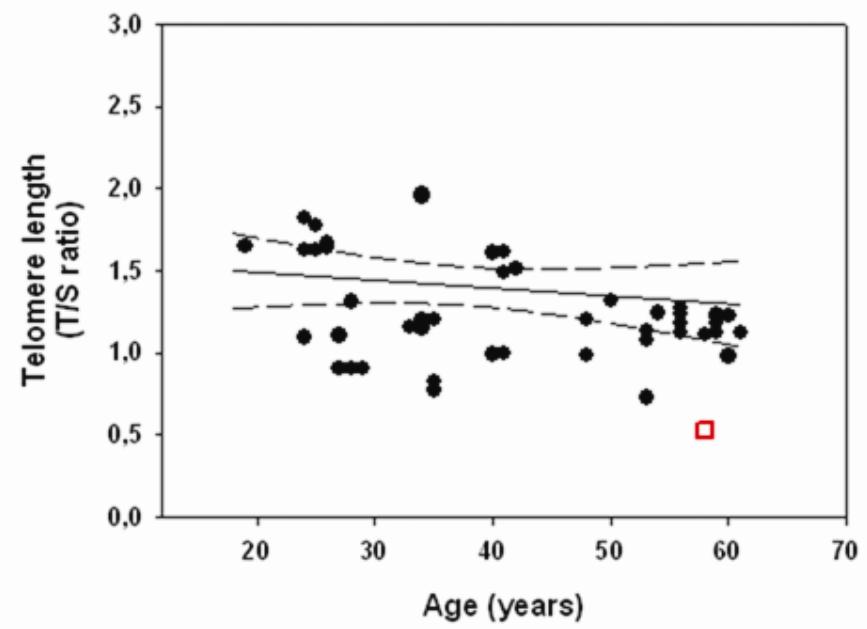

Figure 3. Scatterplot of leukocyte telomere length. The scatterplot, in Figure 3, shows the leukocyte telomere length (LTL) distribution in relation to age in our patient (red square) and in the group of healthy subjects ( black dots). The LTL in our patient is greatly shorter compared to age matched healthy controls. 


\subsection{Telomerase activity}

As shown in Figure 4 telomerase activity of our patient was greatly reduced compared to the control group and also compared to the negative control as well as the positive control supplied with the TRAP Kit (see Figure 4).

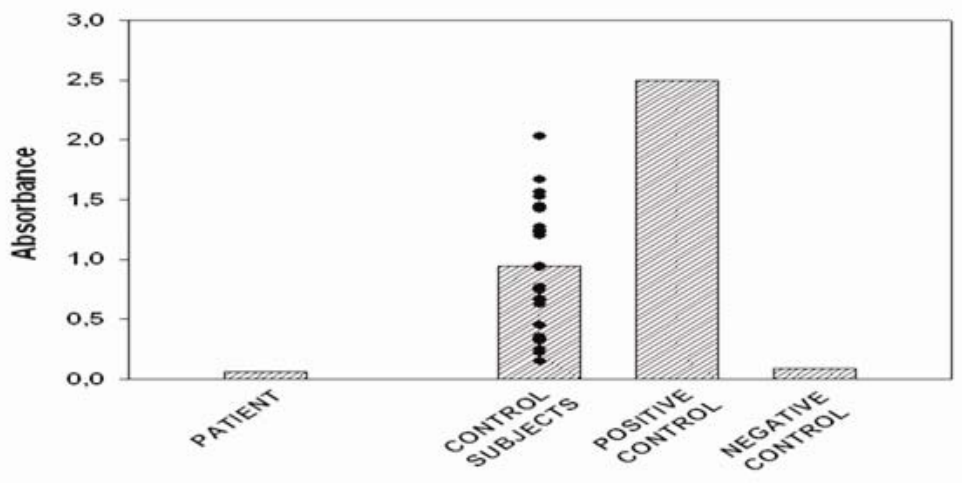

Figure 4. Telomerase activity in our patient and control subjects. As shown in Figure 4, telomerase activity of our patient was greatly reduced compared to the control group and also compared to the negative and positive control supplied with the TRAP Kit.

\subsection{Telomerase mutations}

Genes sequencing did not show any hTERT and hTERC mutations.

\section{Discussion}

The subject we described had synchronous diagnosis of pulmonary fibrosis, NASH-cirrhosis and thrombocytopenia with bone marrow fibrosis.

The clinical presentation of the patient led us to hypothesize the presence of a short telomere syndrome, characterized by the association of multiple organ failure (lung, liver, bone marrow).

The phenotypic heterogeneity caused by mutant telomere complex genes may initially give the impression that telomere shortening causes isolated cases of pulmonary fibrosis, aplastic anaemia or liver disease. However, affected individuals often have subclinical disease concurrently in other organs, even when symptoms related to a single disorder predominate ${ }^{[16]}$. For example, patients with pulmonary fibrosis who have mutant telomerase genes are at an increased risk of developing bone marrow failure and liver disease ${ }^{[13,17]}$ as well as individuals with aplastic anemia have an increased incidence of pulmonary fibrosis when they are exposed to pulmonary toxic drugs in the bone marrow transplant setting, even though they may have previously had no symptom ${ }^{[17,18]}$. Indeed, the co-occurrence of pulmonary fibrosis and bone marrow failure, along with liver cirrhosis, is specific to and highly predictive of a germline telomere maintenance defect $^{[16,17]}$.

The shared underlying telomere defect in aplastic anaemia, pulmonary fibrosis and liver cirrhosis brings together clinical entities that were previously considered to be unrelated and defines a recognizable syndrome complex. The consideration of the telomere syndromes as a single spectrum is important for patient management in different clinical settings ${ }^{[6,14]}$.

Liver cirrhosis and lung fibrosis are chronic, progressive diseases with poor prognosis that share inflammation and fibrosis of target organs as common pathogenic mechanisms. Telomere shortening, deriving from genetic and environmental 
factors, determines cellular senescence and predisposes to multi organ failure and fibrosis. It is well known that pulmonary fibrosis is a disease of telomere maintenance ${ }^{[12]}$.

Recently, two studies have also investigated the frequency of telomerase mutations in patients with sporadic cirrhosis compared to healthy controls ${ }^{[19,20]}$.

These studies screened patients for variation in the TERT and TERC genes. In both studies the frequency of TERT gene mutations in cirrhotic patients was significantly greater than controls. Moreover cirrhosis was also associated with shorter telomeres in peripheral blood leukocytes. Finally, most TERT variants showed reduced telomerase activity in vitro.

Our group was the first to report on the coexistence of cryptogenic cirrhosis (CC) and pulmonary fibrosis and telomere dysfunction associated to the presence of a heterozygous TERT mutation (L153M). Furthermore, leukocyte telomere length was significantly shorter. Our case report not only confirmed the association between pulmonary fibrosis and telomere dysfunction, but, more interestingly, gives further evidence of telomere involvement in liver disease progression and suggests a possible link between NAFLD and CC through telomere shortening ${ }^{[15]}$. Taken together these data show the relation between telomerase mutation and chronic liver disease progression to cirrhosis, probably due to a reduced telomerase activity and therefore an impaired telomere length maintenance ${ }^{[21]}$.

This is a report on a short telomere syndrome involving lung, liver and bone marrow, associated to very short telomere as well very low/absent telomerase activity not in the setting of dyskeratosis congenita.

Sequencing and mutation analysis of hTERT and hTR carried out in our patient did not demonstrated the presence of any mutation. Most likely the telomere dysfunction is due to the involvement of other components of the telomere system.

Probably looking for telomerase genes mutations only, there is the risk to underestimate the real contribution of the telomere system dysfunction to the development of cirrhosis. In fact other components of the telomere complex such as dyskerin and the telomere binding proteins have been shown to be important for telomerase activity ${ }^{[22]}$, mutations in these components can lead to an impairment of telomere function; recently a mutation of the binding protein TIN2 has been involved in the evolution of aplastic anemia ${ }^{[23]}$. Finally also the mutations in the noncoding sequence of TERC and TERC could be responsible for impairment in the expression of TERC and TERT. Probably the sequence analysis of all components of the "telomere system" will reveal the real contribution of telomere complex genes mutations to the development of liver cirrhosis.

Considering telomere disease as a multiple "hits" process, in which short telomeres genetically determined would represent the first step, other environmental stressors accumulating with aging act as additional hits that ultimately provoke what appears to be irreversible multiple organ failure ${ }^{[7]}$. It is intriguing to suggest that in our patient this multi-step progression started with the genetic predisposition most likely due to a telomere complex gene mutation and went on through additional injuries to lung and liver, determined by smoking habits, NAFLD and obesity.

It would have been interesting to evaluate LTL, TA and hTERT, hTERC genes mutations in the other family members since there was a prevalence of NAFLD and overweight in the family.

\section{Conclusion}

Studies with a large number of patients affected by short telomere syndrome are needed to evaluate the real rate of association of clinical or subclinical diseases involving liver, lung and bone marrow with the prevalence of telomerase mutations and to understand the clinical impact of syndromes of telomeres shortening. 
No specific treatment for short telomere syndrome is present nor can be foreseen; treatment only consists in the therapy and follow up of liver cirrhosis and lung fibrosis and eventually a combined transplant. Nonetheless the pathophysiological impact of this condition needs to be underlined.

Patients with telomerase mutations and features of telomeres shortening syndrome may be appropriate targets for future molecular therapies and candidates for educational and surveillance programs (for instance, lifestyle suggestions such as smoking cessation and reduction of alcohol consumption). A thorough personal and family history in individuals with these features is essential for making decisions about diagnostic work-ups, therapeutic options as well as for appropriate genetic counseling. That short telomeres mediate inflammation and fibrosis provides a rationale for pursuing translational strategies aimed at preventing telomere shortening or its cellular consequences as a therapeutic approach

\section{References}

[1] Blackburn EH. Switching and signaling at the telomere. Cell. 2001; 106: 661-73. http://dx.doi.org/10.1016/S0092-8674(01)00492-5

[2] Greider CW, Blackburn EH. Identification of a specific telomere terminal transferase activity in Tetrahymena extracts. Cell. 1985; 43: 405-13.

[3] Armanios M. Telomerase and idiopathic pulmonary fibrosis. Mutat Res. 2012; 730: 52-58. http://dx.doi.org/10.1016/j.mrfmmm.2011.10.013

[4] Armanios M, Chen JL, Chang YP, et al. Haploinsufficiency of telomerase reverse transcriptase leads to anticipation in autosomal dominant dyskeratosis congenita. Proc Natl Acad Sci USA. 2005; 102: 15960-15964. http://dx.doi.org/10.1073/pnas.0508124102

[5] Dokal I. Dyskeratosis congenita. A disease of premature ageing. Lancet. 2001; 358: suppl.1: S22. http://dx.doi.org/10.1016/S0140-6736(01)07040-4

[6] Carulli L, Anzivino C. Telomere and telomerase in chronic liver disease and hepatocarcinoma. In press-W J Gatsroenterol.

[7] Calado RT, Young NS. Telomere diseases. N Engl J Med. 2009; 361: 2353-65.27.

[8] Armanios M. Syndromes of telomere shortening. Annu Rev Genomics Hum Genet. 2009; 10: 45-61. http://dx.doi.org/10.1146/annurev-genom-082908-150046

[9] Yamaguchi H, Calado RT, Ly H, et al. Mutations in TERT, the gene for telomerase reverse transcriptase, in aplastic anemia. N Engl J Med. 2005; 14: 1413-24. PMid:15814878

[10] Knight SW, Heiss NS, Vulliamy TJ, et al. Apastic anaemia, immunodeficiency, and cerebellar hypoplasia (Hoyeraal-Hreidarsson syndrome) due to mutations in the dyskeratosis congenita gene, DKC1. Br J Haematol. 1999; 107: 335-339. http://dx.doi.org/10.1046/j.1365-2141.1999.01690.x

[11] Vulliamy T, Marrone A, Dokal I, et al. Association between aplastic anaemia and mutations in telomerase RNA. Lancet. 2002; 359: 2168-2170. http://dx.doi.org/10.1016/S0140-6736(02)09087-6

[12] Armanios MY, Chen JJ, Cogan JD, et al. Telomerase mutations in families with idiopathic pulmonary fibrosis. N Engl J Med. 2007; 356: 1317-1326. http://dx.doi.org/10.1056/NEJMoa066157.

[13] Alder JK, Chen JJ, Lancaster L, et al. Short telomeres are a risk factor for idiopathic pulmonary fibrosis. Proc Natl Acad Sci USA. 2008; 105: 13051-13056. http://dx.doi.org/10.1073/pnas.0804280105

[14] Armanios M, Blackburn E. The telomere syndromes. Nat Rev Gen. 2012; 13: 693-70. http://dx.doi.org/10.1038/nrg3246

[15] Carulli L, Dei Cas A, Nascimbeni F. Synchronous cryptogenic liver cirrhosis and idiopathic pulmonary fibrosis: a clue to telomere involvement. Hepatology. 2012 Nov; 56(5): 2001-3. http://dx.doi.org/10.1002/hep.26089

[16] Armanios M. Syndromes of telomere shortening. Annu. Rev. Genom. Hum. Genet. 2009; 10:45-61. http://dx.doi.org/10.1146/annurev-genom-082908-150046

[17] Parry EM, Alder JK, Qi X, et al. Syndrome complex of bone marrow failure and pulmonary fibrosis predicts germline defects in telomerase. Blood. 2011; 117: 5607-5611. http://dx.doi.org/10.1182/blood-2010-11-322149

[18] de la Fuente J, Dokal I. Dyskeratosis congenita: advances in the understanding of the telomerase defect and the role of stem cell transplantation. Pediatr. Transplant. 2007; 11: 584-594. http://dx.doi.org/10.1111/j.1399-3046.2007.00721.x

[19] Calado RT, Brudno J, Mehta P, et al. Constitutional telomerase mutations are genetic risk factors for cirrhosis. Hepatology. 2011; 53: 1600-7. http://dx.doi.org/10.1002/hep.24173

[20] Hartmann D, Srivastava U, Thaler M, et al. Telomerase gene mutations are associated with cirrhosis formation. Hepatology. 2011; 53: 1608-1617. http://dx.doi.org/10.1002/hep.24217 
[21] Chaiteerakij R, Roberts LR. Telomerase Mutation: A Genetic Risk Factor for cirrhosis. Hepatology. 2011; 53: 1430-1432. http://dx.doi.org/10.1002/hep.24304

[22] Mitchell JR, Wood E, Collins K. A telomerase component is defective in the human disease dyskeratosis congenita. Nature. 1999; 402: 551-555. http://dx.doi.org/10.1038/990141

[23] Walne AJ, Vulliamy T, Beswick R, et al. TINF2 mutations result in very short telomeres: analysis of a large cohort of patients with dyskeratosis congenita and related bone marrow failure syndromes. Blood. 2008; 112: 3594-3600.

http://dx.doi.org/10.1182/blood-2008-05-153445 